\title{
Summary of ORSphere critical and reactor physics measurements
}

\author{
Margaret A. Marshall ${ }^{\mathrm{a}}$ and John D. Bess \\ Nuclear Science and Technology, Idaho National Laboratory, Idaho Falls, Idaho, USA
}

\begin{abstract}
In the early 1970s Dr. John T. Mihalczo (team leader), J.J. Lynn, and J.R. Taylor performed experiments at the Oak Ridge Critical Experiments Facility (ORCEF) with highly enriched uranium (HEU) metal (called Oak Ridge Alloy or ORALLOY) to recreate GODIVA I results with greater accuracy than those performed at Los Alamos National Laboratory in the 1950s. The purpose of the Oak Ridge ORALLOY Sphere (ORSphere) experiments was to estimate the unreflected and unmoderated critical mass of an idealized sphere of uranium metal corrected to a density, purity, and enrichment such that it could be compared with the GODIVA I experiments. This critical configuration has been evaluated. Preliminary results were presented at ND2013. Since then, the evaluation was finalized and judged to be an acceptable benchmark experiment for the International Criticality Safety Benchmark Experiment Project (ICSBEP). Additionally, reactor physics measurements were performed to determine surface button worths, central void worth, delayed neutron fraction, prompt neutron decay constant, fission density and neutron importance. These measurements have been evaluated and found to be acceptable experiments and are discussed in full detail in the International Handbook of Evaluated Reactor Physics Benchmark Experiments. The purpose of this paper is to summarize all the evaluated critical and reactor physics measurements evaluations.
\end{abstract}

\section{Introduction}

In the early 1970 s Dr. John T. Mihalczo (team leader), J.J. Lynn, and J.R. Taylor performed experiments at the Oak Ridge Critical Experiments Facility (ORCEF) with highly enriched uranium (HEU) metal (called Oak Ridge Alloy or ORALLOY) to recreate GODIVA I results with greater accuracy than those performed at Los Alamos National Laboratory in the 1950s. The purpose of the Oak Ridge ORALLOY Sphere (ORSphere) experiments was to estimate the unreflected and unmoderated critical mass of an idealized sphere of uranium metal corrected to a density, purity, and enrichment such that it could be compared with the GODIVA I experiments. In addition to two critical configurations, reactor physics measurements were also performed [1-7]. The critical configuration and reactor physics measurements have been evaluated and accepted for inclusion in the International Handbook of Evaluated Criticality Safety Benchmark Experiments (ICSBEP Handbook) and the International Handbook of Evaluated Reactor Physics Benchmark Experiments (IRPhEP Handbook) $[8,9]$. Preliminary results of the critical evaluation were given at Nuclear Data 2013 [10]. Final results of the critical evaluation and the reactor physics evaluations are given here.

\section{Experiment overview}

The ORSphere was an HEU metal sphere that was originally used for neutron leakage measurements by General Atomic Company. In order to take what was a subcritical system and create the critical ORSphere, the

a e-mail: margaret.marshall@inl.gov
5 part sphere was made into a 3 part sphere using HEU pins, and the target hole and thermocouple groove were filled. A general schematic of the ORSphere is given in Fig. 1(a). The first critical configuration had an average radius of $8.80491-\mathrm{cm}$ (3.4665-inches) and an assembled reactivity of $68.1 \pm 2.0 \notin$. The sphere was then machined to 8.74268 -cm-average-radius (3.4420-inch). The reactivity of the smaller sphere was $-23.4 \phi$. The change between the two sphere configurations gives a surface material worth of $0.086 \phi$ per gram of surface material.

Buttons were used on the surface of the sphere to adjust system reactivity. Various thicknesses and types of buttons were measured. The worth of the diametral rod was also measured. Additionally, the center place was re-machined to allow for a central void measurement; see Fig. 1(b).

Kinetic parameters $\beta_{\text {eff }}, \Lambda$, and $\alpha$ were also derived using experimental data for the ORSphere. Finally, the fission density and neutron importance was measured through the center of the sphere. Any additional measurements made by the experimenter were not evaluated.

\section{Experiment evaluation}

The experiments were evaluated for inclusion in the ICSBEP and IRPhEP Handbooks. Monte Carlo N-Particle (MCNP) Version 51.60 and ENDF/B-VII.0 neutron cross section libraries were used for the evaluation. The effect of measurement, geometric and material uncertainties on the experiment measurements were evaluated. Benchmark models were created and simplification biases were derived.

(C) The Authors, published by EDP Sciences. This is an Open Access article distributed under the terms of the Creative Commons Attribution License 4.0 (http://creativecommons.org/licenses/by/4.0/). 


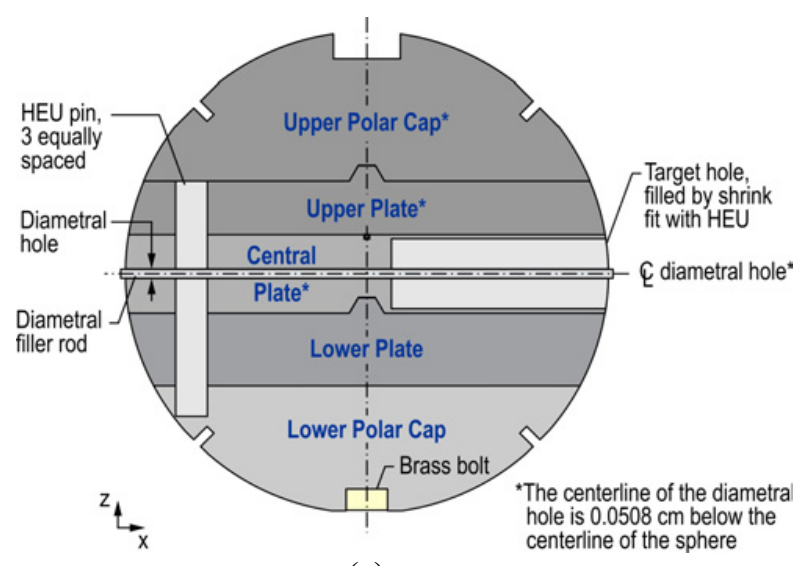

(a)

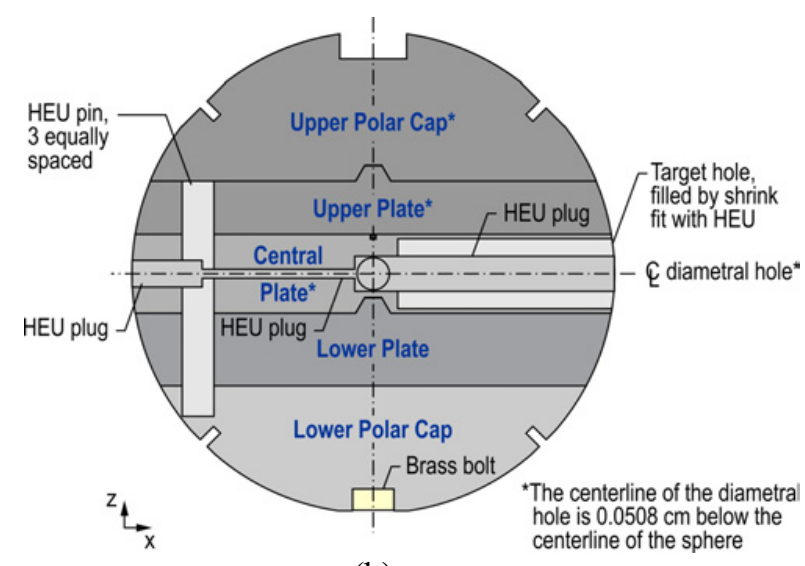

(b)

Figure 1. Schematic of the ORSphere Assembly. (a) critical configuration. (b) kinetic parameter and central void measurement configuration.

Table 1. Benchmark Experiment Eigenvalues.

\begin{tabular}{|lcc|}
\hline $\begin{array}{l}\text { Benchmark } \\
\text { Model } k_{\text {eff }}\end{array}$ & Case 1 & Case 2 \\
\hline Detailed & $1.0026 \pm 0.0007$ & $0.9966 \pm 0.0007$ \\
\hline Simple & $1.0031 \pm 0.0007$ & $0.9966 \pm 0.0007$ \\
\hline
\end{tabular}

\subsection{Critical and worth measurements}

The total experimental uncertainty for the slightly super- and slightly sub-critical sphere was 0.00071 and $0.00070 \Delta \mathrm{k}_{\mathrm{eff}}$, respectively. The measurement uncertainty contributed about 0.00014 and $0.00013 \Delta \mathrm{k}_{\text {eff }}$. The major contributor to the experimental uncertainty was the uncertainty in the curve of the ellipsoidal parts. The spherecity of each part of the sphere was measured at a finite number of points using a sweep gage. The finite number of measured points leads to an uncertainty in the curvature of the pieces. This is manifest in a density difference between the two experimental cases. The effect of this uncertainty is $0.00066 \Delta \mathrm{k}_{\mathrm{eff}}$.

Detailed and simple benchmark models were created for the two critical configurations. The detailed model had all details modelled within the sphere itself but with the surrounding structure and air removed. The simplification bias was $-0.00192 \pm 0.00013 \Delta \mathrm{k}_{\mathrm{eff}}$ and $-0.00191 \pm$ $0.00013 \Delta \mathrm{k}_{\mathrm{eff}}$ for Case 1 and 2 respectively. The simple benchmark model was a homogenized sphere with only three impurities present. Si, B, and $\mathrm{C}$ were included in the simple benchmark model because of the large bias associated with removing them. The bias of the simple benchmark model was $0.00140 \pm 0.0013$ and $-0.00185 \pm$ $0.00013 \Delta \mathrm{k}_{\mathrm{eff}}$ for Case 1 and 2 , respectively. The resulting benchmark eigenvalues are given in Table 1 .

The reactivity of the system is determined using a stable reactor period measurement. A modified version of the Inhour equation, using the Keepin et al. delayed neutron parameters, is used to convert the stable reactor periods to reactivity in units of dollars. Worth measurements were the difference between the system reactivities with and without a change in the system. For example, the central void worth was measured as the difference in the system reactivity with and without a small, uranium sphere present at the center of the
ORSphere. The worth of the central void was $9.165 \pm$ $0.023 \phi$.

The worth of buttons was measured with a similar method. Various numbers of uranium, aluminum, and stainless steel buttons were placed on the surface of the sphere and in the empty socket hole of the upper polar cap. The buttons were $2.21488 \mathrm{~cm}$ in diameter, with an inner diameter of $0.44958 \mathrm{~cm}$, and thicknesses of $0.3175 \mathrm{~cm}$ and $0.635 \mathrm{~cm}$.

The effect of uncertainty was evaluated for the center void and button worth measurements. The effect of measurement and the inhour equation methods, dimension, and material uncertainties were evaluated. The largest contributions to the experimental uncertainty were the measurement uncertainty and the uncertainty in the delayed neutron parameters. If a high number of stable reactor period measurements were performed, as was done for the central void worth, the measurement uncertainty was decreased. But for some of the button worth measurements a limited number of stable reactor period measurements were performed and resulted in a higher measurement uncertainty. The experimenter used Keepin delayed neutron parameters [11] when converting the stable reactor period to a system reactivity. The effect of the systematic uncertainty in these delayed neutron parameters contributed to a $1.32 \%$ uncertainty in the worth measurement. The effect of biases was also evaluated and found to be negligible. The benchmark worth measurements are summarized in Table 2.

\subsection{Kinetic parameters}

Because the central void reactivity was found independent of $\beta_{\mathrm{eff}}$, the ratio of the reactivity in units of $\Delta \mathrm{k}_{\mathrm{eff}}$ to reactivity in units of dollars gives $\beta_{\text {eff. The central void }}$ reactivity measurement is in units of dollars. The value was calculated using $S_{n}$ discrete-ordinate transport theory in units of $\Delta \mathrm{k}_{\text {eff }}$. This method is similar to one used by Gordon Hansen [12]. Initially, the $\Delta \mathrm{k}_{\text {eff }}$ reactivity was calculated using ONEDANT and XSDRNPM codes with Hansen-Roach and ENDF/B-VI cross section libraries were used. During the evaluation process the value was re-calculated and differences were accounted for as uncertainty. The benchmark central void worth was $9.165 \pm 0.123 \notin$. The worth was calculated to be 
Table 2. Benchmark worth values.

\begin{tabular}{|c|c|}
\hline & Worth $(\phi)$ \\
\hline Central Void & $9.165 \pm 0.123$ \\
\hline $\begin{array}{l}16,0.635-\mathrm{cm} \text {-thick Uranium Buttons } \\
\text { on Sphere Surface }\end{array}$ & $35.4 \pm 2.868$ \\
\hline $\begin{array}{l}4,0.3175-\mathrm{cm} \text {-thick Uranium Buttons } \\
\text { on Sphere Surface }\end{array}$ & $6.1415 \pm 0.117$ \\
\hline $\begin{array}{l}1,0.3175 \text {-cm-thick Aluminum Button } \\
\text { on Sphere Surface }\end{array}$ & $0.7058 \pm 0.062$ \\
\hline $\begin{array}{l}3,0.3175-\mathrm{cm} \text {-thick Uranium Buttons } \\
\text { in Socket Hole }\end{array}$ & $7.86 \pm 0.127$ \\
\hline $\begin{array}{l}3,0.3175 \text {-cm-thick Stainless Steel Buttons } \\
\text { in Socket Hole }\end{array}$ & $4.7228 \pm 0.076$ \\
\hline $\begin{array}{l}3,0.3175-\mathrm{cm} \text {-thick Aluminum Buttons } \\
\text { in Socket Hole }\end{array}$ & $3.1259 \pm 0.074$ \\
\hline Diametral Filler Rod & $11.234 \pm 0.168$ \\
\hline
\end{tabular}

$6.02 \pm 0.023 \times 10^{-4}$. This yielded a benchmark worth of $0.00657 \pm 0.00009 \beta_{\text {eff }}$. It was determined that this method is only valid for systems of uniform composition whose worth can be accurately calculated.

In addition to $\beta_{\text {eff }}$, the prompt neutron decay constant is measured and the mean neutron generation time is derived for the ORSphere. The prompt neutron decay constant, $\alpha$, was measured using Rossi- $\alpha$ and randomly pulsed neutron measurements. 538 measurements in seven detector and source location configurations were measured. Data was fit using non-linear least squares regression to get an $\alpha$ value of $1.1061 \pm 0.0009 \mu \mathrm{s}^{-1}$. This uncertainty was based on the statistics of all 538 measurements. Because of correlation this would be an underestimate of the true uncertainty. The experimental uncertainty was increased to $\pm 0.0079 \mu \mathrm{s}^{-1}$ which accounts for the fact that only seven unique configurations were used. An additional bias uncertainty had to be considered for the benchmark model because the sphere was brought to delayed critical for the $\alpha$ measurements using uranium buttons. However, the exact details of button use was not specified. The benchmark model for the slightly subcritical sphere was used and mass was added to the sphere using the benchmark surface mass coefficient to bring the system to $k=1$. This added a rather large bias uncertainty. The resulting benchmark value is $1.1061 \pm 0.1071 \mu \mathrm{s}^{-1}$.

Because $\beta_{\text {eff }}$ and $\alpha$ were measured the mean neutron generation time, $\Lambda$, can be derived using the following equation.

$$
\alpha=\frac{\rho-\beta_{\text {eff }}}{\Lambda}
$$

Using the experimental values and experimental uncertainties of $\beta_{\text {eff }}=0.00657 \pm 0.00009, \rho=0 \pm 2 \phi, \alpha=$ $-1.1061 \pm 0.0079 \mu \mathrm{sec}^{-1}$, an experimental $\Lambda$ value of $0.00594 \mu \mathrm{sec}$ or 5.94 nanosec is derived. The experimental uncertainties can be propagated to obtain an uncertainty of \pm 0.15 nanosec.

\subsection{Reaction rate measurements}

Neutron importance and fission density were measured through the center of the ORSphere. The idea of neutron importance was to determine the effect on the external neutron population of a neutron born at a specified radial location within the sphere. It was measured by passing a Cf source through the diametral hole of the sphere and

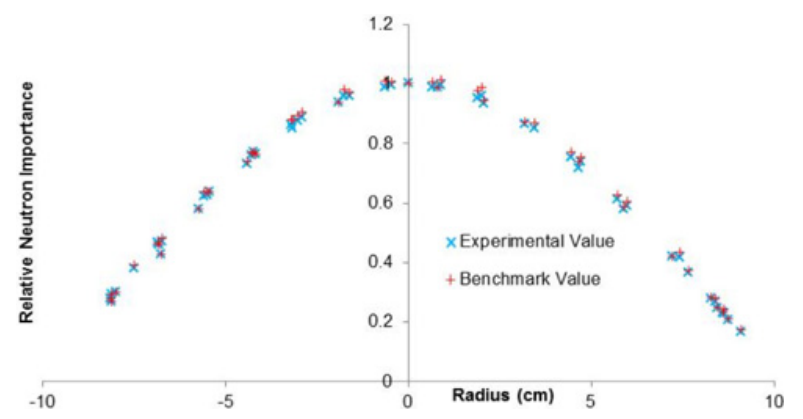

Figure 2. Relative Neutron Importance.

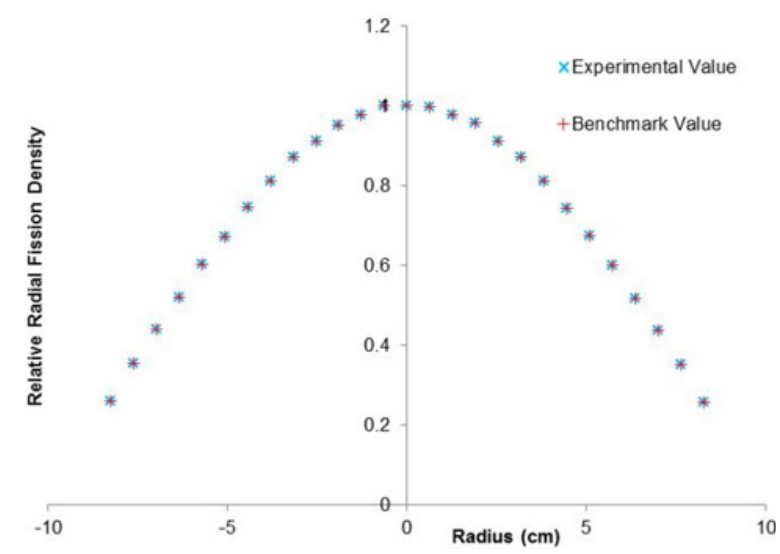

Figure 3. Relative fission density.

neutron population was monitored using three detectors external to the sphere. The benchmark uncertainty was less than $2 \%$ for all data points except one. This uncertainty included the measurement uncertainty given by the experimenter, geometric, and material uncertainties as well as rounding and normalization uncertainties. The one outlying point has an approximately $10 \%$ uncertainty, as given by the experimenter, for unknown reasons. The simplification bias for the neutron importance was quite small or negligible. Experimental and benchmark results are given in Fig. 2.

Fission density was measured using cylinders of uranium which were placed in the diameteral hole of the ORSphere and were activated. The resulting fission product gamma-ray activity was measured with a pair of NaI scintillation detectors. A normalization cylinder which was at the center of the sphere during activation was counted simultaneously. The benchmark uncertainty was approximately $1 \%$ or less. The simplification bias for the relative fission density was negligible or very small. The experimental and benchmark fission density is shown in Fig. 3.

\section{Sample calculations and conclusions}

Sample calculations were performed and reported for the measurements. Various codes and cross section libraries were used. For the critical configurations sample calculation results range from about $0.5 \%$ high to $0.3 \%$ low for the various codes for the detailed benchmark model.

For the worth measurements, the sample calculations varied by as much as $25 \%$ however, it should be noted that due to high benchmark uncertainty and/or statistical uncertainty of the calculations this calculation bias is not as drastic as it may seem. 
Table 3. Benchmark worth values for $\alpha$ and $\Lambda$.

\begin{tabular}{|ll|ll|ll|}
\hline $\mathrm{k}_{\text {eff }}$ & $1.00141 \pm 0.00002$ & Benchmark $\alpha(\mu \mathrm{s})$ & $-1.1061 \pm 0.1071$ & Benchmark $\Lambda(\mathrm{ns})$ & $5.94 \pm 0.014$ \\
\hline$\beta_{\text {eff }}$ & $0.00647 \pm 0.00003$ & Calculated $\alpha(\mu \mathrm{s})$ & $-0.8842 \pm 0.0902$ & Calculated $\Lambda(\mathrm{ns})$ & $5.72499 \pm 0.00297$ \\
\hline$\Lambda(\mathrm{ns})$ & $5.72499 \pm 0.00297$ & $(C-E) / E^{(\mathrm{a})}$ & $-20 \%$ & $(C-E) / E^{(\mathrm{a})}$ & $-3.6 \%$ \\
\hline
\end{tabular}

(a) "E" is the expected or benchmark value. "C" is the calculated value.

Using MCNP5 and ENDF/B-VII.0 the $\beta_{\text {eff }}$ calculates to be 0.00654 , which is about $1 \%$ low, using adjointweighted method capabilities. The prompt neutron decay constant was calculated using adjoint-weighted method capabilities in MCNP5-1.60. By calculating $\mathrm{k}_{\mathrm{eff}}, \beta_{\mathrm{eff}}$, and $\Lambda$ in MCNP $\alpha$ is then calculated. The results are given in Table 3. It should be noted that the benchmark model for $\alpha$ and $\Lambda$ is different from the benchmark model for $\beta_{\text {eff. The }}$ benchmark model for $\alpha$ has a $\mathrm{k}_{\mathrm{eff}}$ of 1 . The benchmark model is calculating approximately $0.14 \%$ high. It is of interested to note that, if the calculational bias is ignored and the reactivity is set to zero the calculated $\alpha$ becomes $-1.1301 \mu \mathrm{sec}$ which is only $2 \%$ above the benchmark value.

The ORSphere benchmarks are included in the International Handbook of Evaluated Criticality Safety Benchmark Experiments (ICSBEP Handbook) and the International handbook of Evaluated Reactor Physics Benchmark Experiments (IRPhEP Handbook) $[8,9]$. The critical measurement and all reactor physics measurements are judged to be acceptable as benchmark experiments. They can be used for code and cross section validation. It should be noted that the evaluations from the latest version of the handbooks should always be referenced for the most up-to-date information.

\section{References}

[1] J. Mihalczo, J. Lynn, J. Taylor, G. Hansen, D. Pelowitz, Ann. Nucl. Energy 29, 525-560 (2002)

[2] J.T. Mihaclzo, J.J. Lynn, J.R. Taylor, and G.E. Hansen, "Measurements with an Unreflected Uranium (93.2\%) Metal Sphere," PHYSOR 1993, Nashville, TN, September 19-23 (1993)

[3] J.T. Mihalczo, J.J. Lynn, and J.R. Taylor, Nuc. Sci. and Eng. 130, 153-163 (1998)

[4] J.T. Mihalczo, J.J. Lynn, and J.R. Taylor, ORNL/TM13349 (1997)

[5] J.T. Mihalczo, Nucl. Sci. Eng. 60, 262-275 (1976)

[6] J.T. Mihalczo, Nucl. Tech. 175, 498-508 (2011)

[7] J.T. Mihalczo, Nucl. Sci. Eng. 56, 271-290 (1975)

[8] M.A. Marshall, ICSBEP Handbook, HEU-METFAST-100 (2013)

[9] M.A. Marshall, IRPhEP Handbook, ORSPHEREFUND-EXP-001 (2014)

[10] M. Marshall, J. Bess, and T. Murray, Nucl. Data Sheets 115, 469-471 (2014)

[11] G.R. Keepin, T.F. Wimett, R.K. Zeigler, Phys. Rev. 107, 1044 (1975)

[12] G.E. Hansen and C. Maier, LA-1525 (1953) 